\title{
Tissue response evaluation of the mucosa of the tympanic cavity of guinea pigs, when receiving biodegradable implant ${ }^{1}$
}

\author{
Leandro de Borborema Garcia', Armando da Silva Cunha JuniorII, Silvia Ligório Fialho ${ }^{\mathrm{III}}$, André Coura Perez ${ }^{\mathrm{IV}}$, Bruno Borges \\ de Carvalho Barrosv", Ricardo Schaffeln DoriguetovI, José Ricardo Gurgel Testa ${ }^{\text {VII }}$
}

DOI: http://dx.doi.org/10.1590/S0102-86502014001300003

IFellow Master degree, Medicine (Otolaryngology) Post-Graduate Program - Department of Otorhinolaryngology and Head and Neck Surgery Federal University of São Paulo - Paulista School of Medicine. Substantive scientific and intellectual contributions to the study, conception and design, manuscript preparation, acquisition of data, analysis and interpretation of data, manuscript writing.

IIPhD in Pharmaceutical Sciences - Université de Paris XI (Paris-Sud). Associate Professor of Faculty of Pharmacy, Federal University of Minas Gerais. Conception and design of the study.

IIIPhD in Pharmaceutical Sciences -Federal University of Minas Gerais. Manager Pharmacotechnical Development and Biotechnology of Ezequiel Dias Foundation. Conception and design of the study.

${ }^{\mathrm{IV}} \mathrm{PhD}$ - Medicine (Otolaryngology) - USP - Faculty of Medicine of Ribeirao Preto. Conception and design of the study, manuscript preparation, acquisition of data

vFellow Master Degree -Medicine (Otolaryngology) Post-Graduate Program - Department of Otorhinolaryngology and Head and Neck Surgery Federal University of São Paulo - Paulista School of Medicine. Manuscript preparation; acquisition of data

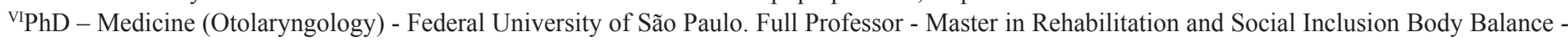
Anhanguera Educacional. Manuscript preparation; acquisition of data.

${ }^{\mathrm{VII}} \mathrm{PhD}$ in Science - UNIFESP; Adjunct Professor at the Department of Otorhinolaryngology and Head and Neck Surgery - UNIFESP-EPM. Medical School of the Federal University of São Paulo - UNIFESP/EPM. Responsible for conception and design, manuscript preparation, critical revision, final approval.

\section{ABSTRACT}

PURPOSE: To evaluate the tissue response of the mucosa of the tympanic cavity of guinea pigs, when receiving biodegradable implant. METHODS: A total of 20 male guinea pigs were divided into 2 groups. After paracentesis in both ears, a biodegradable polymer of poly lactic-co-glycolic acid was implanted in only one middle ear. Histological analysis using neutrophil exudate and vascular neoformation (acute inflammation) and fibroblast proliferation and mononuclear inflammatory cells (chronic inflammation) as parameters was performed after 10 and 30 days of survival (groups 1 and 2, respectively).

RESULTS: Four ears in group 1 and 7 in group 2 had an increase of neutrophil exudate. Vascular neoformation occurred in ears with or without the implant, in both groups. Fibroblast proliferation and mononuclear inflammatory cells (lymphocytes and macrophages) increased in ears with implant in group 2.

CONCLUSION: The tissue response by histological analysis of the mucosa of the tympanic cavity of guinea pigs, when receiving biodegradable implant, showed no statistically significant difference between ears with or without the implant.

Key words: Biopolymers. Inflammation. Ear, Middle. Guinea Pigs. 


\section{Introduction}

Treatment of cochlear disorders represents a promising future in otology, but the underlying pathophysiology of common diseases such as Menière's syndrome and sensorineural hearing loss remain poorly understood. Therapeutic methods for diseases of the inner ear are available, and these largely depend on the route and the concentrations of the drugs used ${ }^{1}$.

Nowadays, there is an increasing interest in the treatment of human inner ear disorders by local drug delivery to the inner ear for the dual purposes of increasing efficacy and decreasing side effects ${ }^{2-5}$.

The variety of existing local drug delivery strategies to the inner ear include: 1) single or repeated intratympanic injection with or without volume stabilization and with or without visualization of the round window (RW) membrane, 2) continuous or discontinuous drug application via partly or fully implantable pump systems, and 3) biodegradable biopolymers ${ }^{2}$.

Biodegradable polymer, defined as the polymer that suffer macromolecular degradation in vivo, may present advantages against the non-degradable polymer, due its total absorption by the organism and no need of removal6-8.

The most frequently used biodegradable polymers are polyesters such as caprolactone, polylactic acid (PLA) and various lactic and glycolic acid copolymer types (PLGA), the latter two being extensively used ${ }^{7}$. PLGA can be easily produced and has predictable decomposition kinetics. It is degraded by the hydrolysis of its corresponding monomers (lactic and glycolic acids), which are eliminated with minimal systemic toxicity ${ }^{8,9}$.

It is desired that the material has the ability to perform its desired function with respect to a medical therapy, without eliciting any undesirable local or systemic effects in the recipient or beneficiary of that therapy, but generating the most appropriate beneficial cellular or tissue response in that specific situation, and optimizing the clinically relevant performance of that therapy ${ }^{10,11}$ physical and chemical characterization, followed by an in vitro degradation study. Further, $n 0$ and $n 30$ in both virgin and 3-week pre-degraded status were subcutaneously implanted in rats, either directly or in stainless steel mesh cages, to evaluate in vivo tissue response. The results showed that the incorporation of nAp yields an nAp amount-dependent buffering effect on $\mathrm{pH}$ levels during degradation and delayed polymer degradation based on molecular weight analysis. Regarding biocompatibility, nAp incorporation significantly improved the tissue response during a 4-week subcutaneous implantation, showing less infiltration of inflammatory cells (monocyte/macrophages.
Therefore, the aim of this study is to evaluate the tissue response of the mucosa of the tympanic cavity of guinea pigs, when receiving biodegradable implant.

\section{Methods}

The study was accomplished in the bioterium of Institute of Advanced Sciences in Otolaryngology (IASO) and Temporal Bone Microdissection Laboratory - Federal University of Sao Paulo (UNIFESP), after approval by the Ethics and Research Committee of UNIFESP (number 0213/12) and with supervision and monitoring of a veterinary, following the Council for International Organization of Medical Sciences (CIOMS) ethical code for animal experimentation and obeyed the norms of the Federal Law number 6,638/1979.

Twenty guinea pigs (Cavia porcellus), male, 2 to 3 months of age, weighing around $400 \mathrm{~g}$ and normal otomicroscopy were randomly divided into two groups: group $1(n=10)$ with 10 days of survival and group $2(\mathrm{n}=10)$ with 30 days of survival.

The biopolymers were developed in Laboratory of Pharmaceutical Technology - Faculty of Pharmacy, Federal University of Minas Gerais. In the preparation of the biodegradable implants, PLGA was solubilized on proper solvent and distilled water. The solution was then filtered using a sterile $0.2 \mathrm{~mm}$ filter under laminar flow. Next it was frozen dried, and used to prepare hot-molded rod-shaped implants. The implants weighed between 0.9 and $1.2 \mathrm{mg}$, measured 4.5 to $6.0 \mathrm{~mm}$ in length, and had diameters ranging between 0.4 and $0.5 \mathrm{~mm}$. The low coefficient of variation between the implants was indicative of the reproducibility of the employed technique.

The guinea pigs were anesthetized with xylazine hydrochloride $(20 \mathrm{mg} / \mathrm{Kg})$ and ketamine hydrochloride $(10 \mathrm{mg} /$ $\mathrm{Kg}$ ) by peritoneal route, remaining under spontaneous ventilation throughout the procedure.

All the animals were submitted to surgical procedure bilaterally, in agreement with technique to be described. The contralateral ears to the placement of the biopolymers were considered controls.

Afther the anesthesia, the guinea pigs were positioned at the surgical bed and antisepsis of the external ear were made. Then, submitted to otomicroscopy for verification of the normality of external auditory meatus (EAM) and tympanic membrane (TM). Paracentesis in the inferior part of TM was made and then the biopolymer was inserted into middle ear, as near as possible to the round window. In the contralateral ear, after paracentesis, no more manipulation was made. At 
the end of the procedure, all animals received an intracardiac administration of potassium chloride $19.1 \%$, while still anesthetized for euthanasia.

After the euthanasia, both temporary bones were extracted, stored in formaldehyde $10 \%$ and directed for histologic exam. Initially immersed in nitric acid 5\% for 24 hours for decalcification, the temporary bone was cut in the medium line, longitudinally, and submitted to the usual techniques for histologic exam, colored by hematoxilin-eosin (HE).

The acute and chronic inflammatory conditions and the healing status in each ear were appraised. For acute inflammation was evaluated the presence of neutrophils and vascular neoformation and for chronic inflammation the fibrous conjunctive tissue proliferation and inflammatory mononuclear cells.

Software package SPSS (Statistical Package for Social Sciences) release 22.0 was used to process raw data and produce statistical results. Data organization was done with Excel MSOffice 2010. A statistical significance level of 5\% (0.05) was adopted in statistical tests (Likelihood Ratio Test).

\section{Results}

In group 1, exudate of neutrophils was found in 4 ears, increasing this number to 7 in group 2 - five of them in ears with the biopolymer (Table 1). The same assessment occurred for vascular neoformation, with $20 \%$ of this neoformation in ears with the implant in group 1 . This percentage increased to $40 \%$ in group 2. This vascular neoformation was also found in ears without the implant, in both groups (Table 2).

Most of the ears had absence of fibrous conjunctive tissue proliferation, in the two groups. An increase occurred again in ears with the implant in group 2 (Table 3 ).

TABLE 1 - Histologic evaluation - neutrophil exudate.



$\mathrm{p}<0.05$; Test: Likelihood Ratio Test 
TABLE 2 - Histologic evaluation - vascular neoformation.

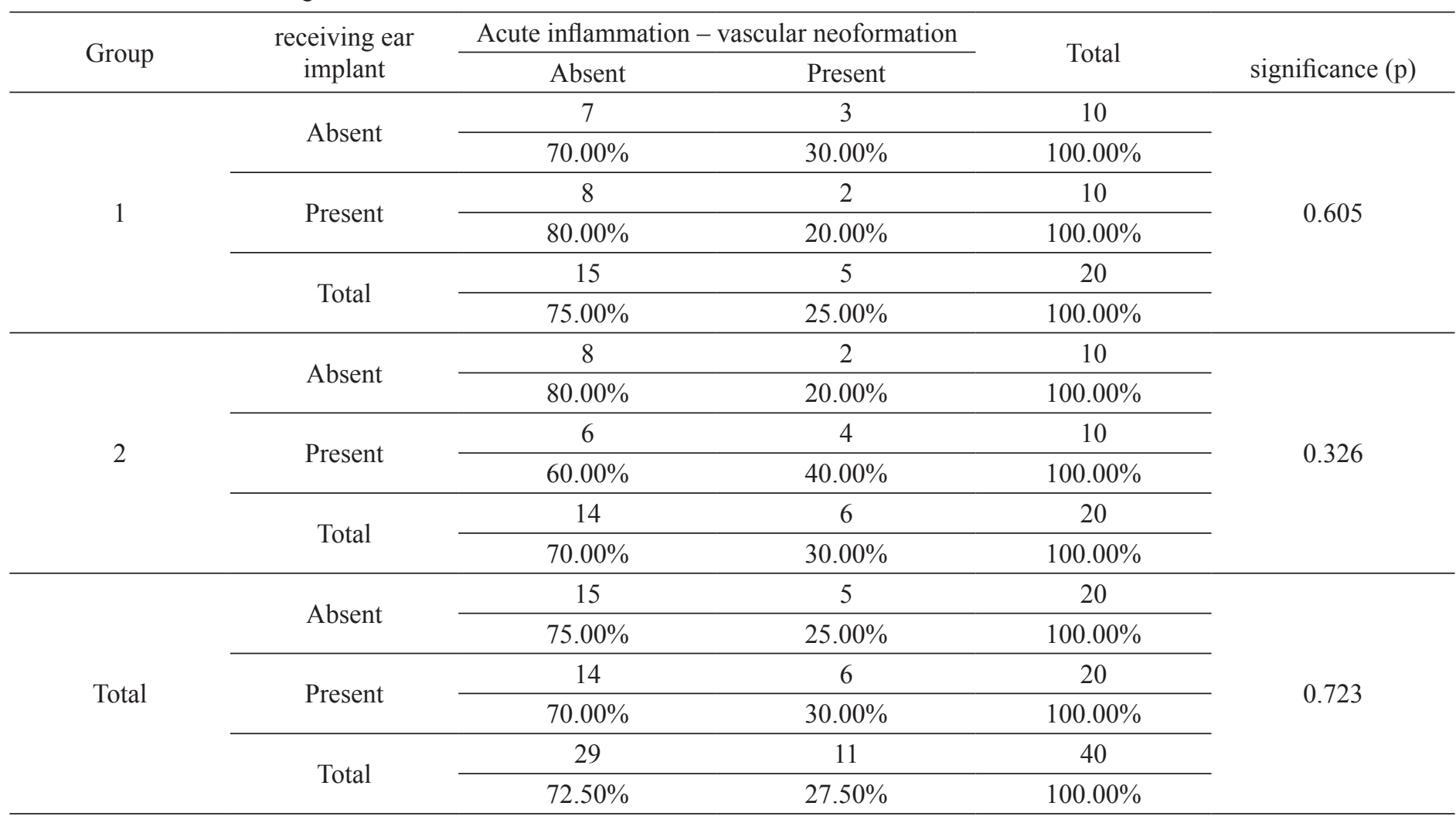

$\mathrm{p}<0.05$; Test: Likelihood Ratio Test

TABLE 3 - Histologic evaluation - fibrous conjunctive tissue proliferation.

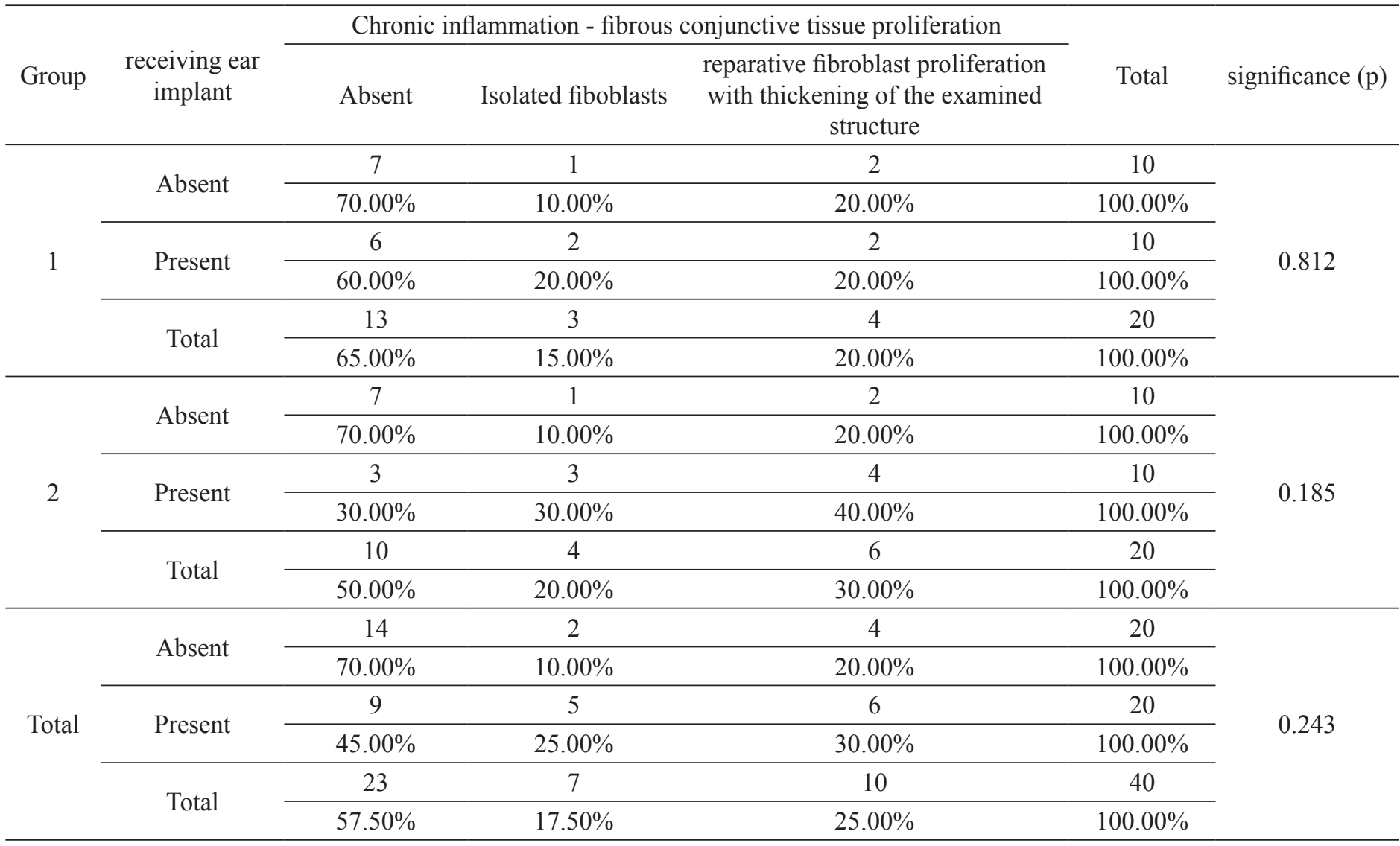

$\mathrm{p}<0.05$; Test: Likelihood Ratio Test 
Garcia LB et al.

Similar numbers were found for inflammatory mononuclear cells (lymphocytes and macrophages). Initially, all the ears of both groups showed at least isolated foci of these cells. In group 1, diffused infiltrate was found in $30 \%$ of the ears with the biopolymer, increasing this percentage to $40 \%$ in group 2 (Table 4) (Figure 1).

TABLE 4 - Histologic evaluation - inflammatory mononuclear cells (lymphocytes and macrophages).

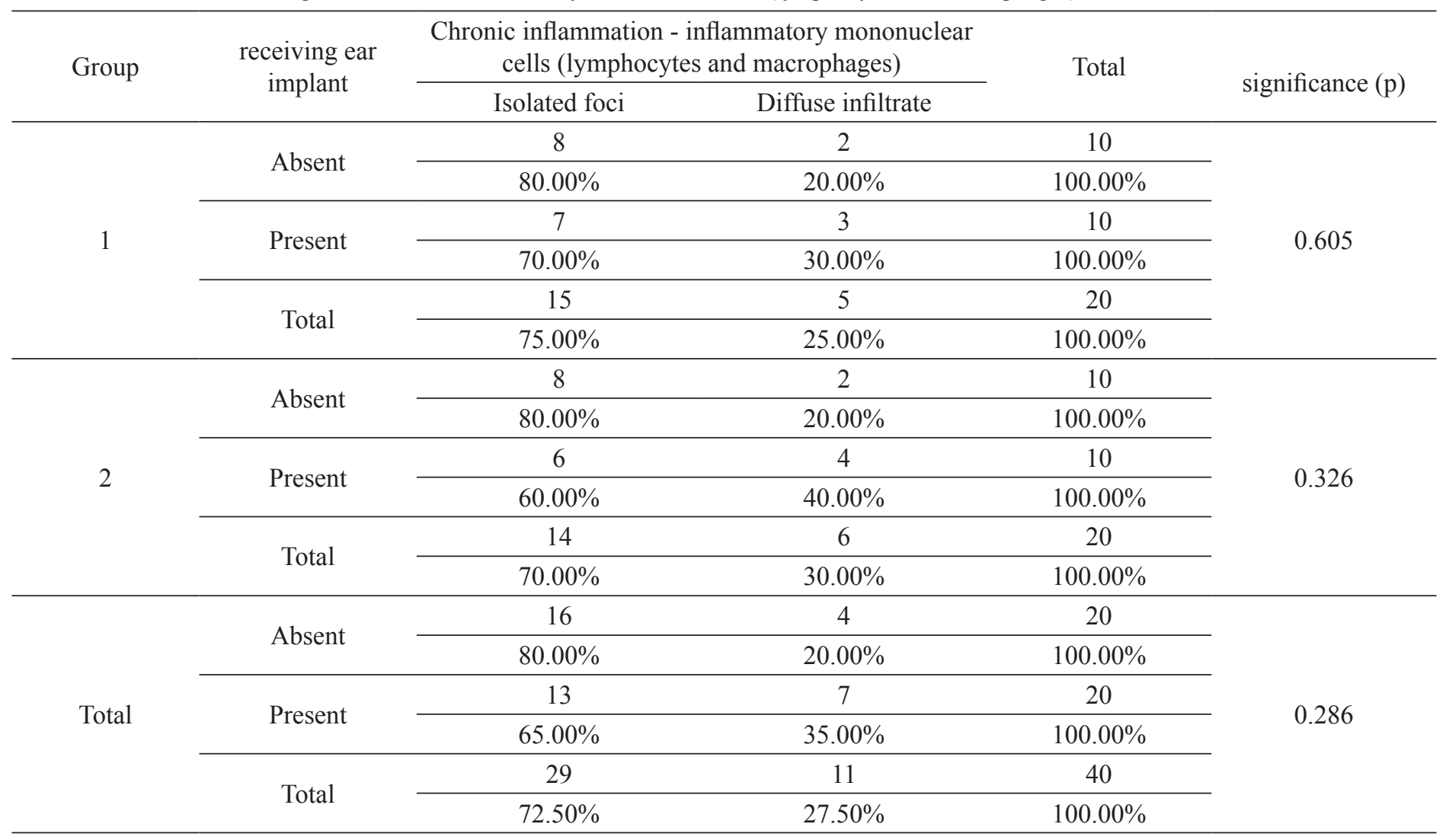

$\mathrm{p}<0.05$; Test: Likelihood Ratio Test

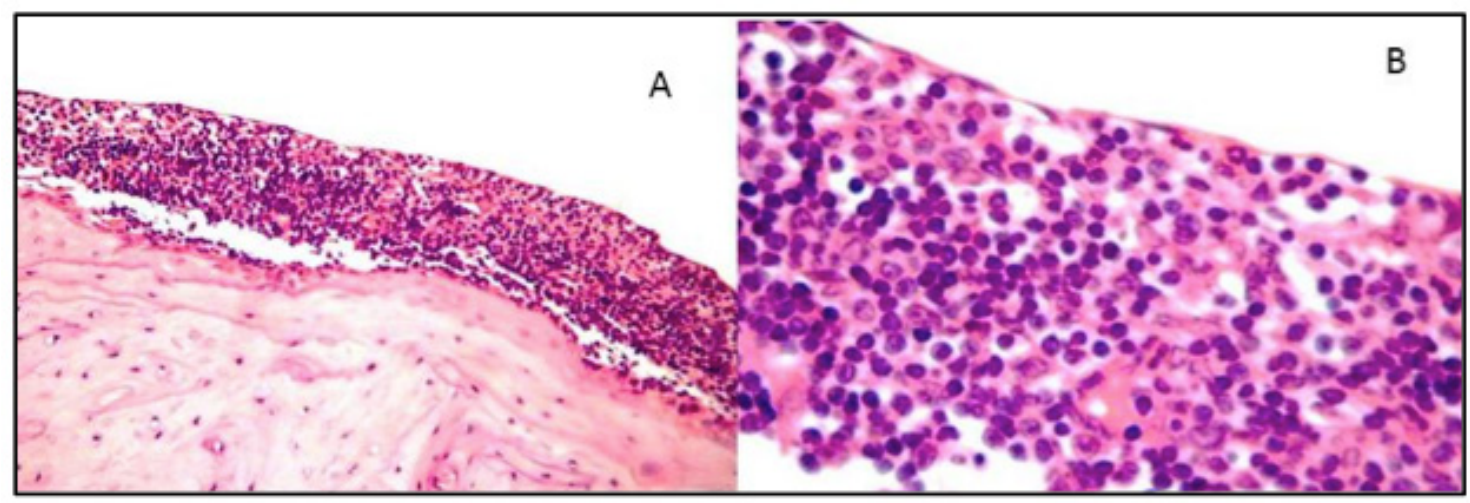

FIGURE 1 - Mucosa of the tympanic cavity of guinea pig number 04 (right ear) with inflammatory mononuclear cells. A: x100 magnification, B: x400 magnification. HE stain. 


\section{Discussion}

Inner ear drug delivery has been a challenge to physicians in the treatment of inner ear disorders. In the past decade, new biomaterials and drug delivery technologies have been developed for inner ear delivery ${ }^{2,5}$.

PLGA was the polymer chosen in this study, because is widely used in studies related to drug delivery systems and is a constituent of many biomaterial-based devices owing to its adjustable biodegradability and biocompatibility ${ }^{10}$. Also, this polymer is easily produced and replicated, and has a well-known kinetic of degradation?.

Several methods exist for the evaluation of the biocompatibility of biopolymers. The method used in the study was the histological analysis with Hematoxylin and eosin stain. According to Kato et al. ${ }^{12}$, it is the more used method in experimental studies.. It is a simple method and supplies general information of the tissue answer to the implanted material.

Host reactions following implantation of biomaterials include injury, blood-material interactions, provisional matrix formation, acute inflammation, chronic inflammation, granulation tissue development, foreign body reaction, and fibrosis/fibrous capsule development. Following the initial blood/material interactions and provisional matrix formation, acute and chronic inflammation occurs in a sequential fashion as expected. The extent or degree of these responses is controlled by the extent of injury in the implantation procedure, the tissue or organ into which the device is implanted, and the extent of provisional matrix formation ${ }^{13}$.

The histologic evaluation of the mucosa of the guinea pigs suggested that the implanted biopolymer was not a cause of acute or chronic inflammation. The study didn `t find any statistical difference in the comparison of the ears that received or not received the biopolymer.

An increase occurred in Group 2 comparing to Group 1, for both neutrophils and neovascularization, in implanted ears, but it has no statistic difference. This result shows a weak relationship between the biopolymer and the parameters of acute inflammation.

When analyzed chronic inflammation, the same results were found. Although again there were an increasing number of implanted ears, from group 1 to group 2, with inflammatory mononuclear cells and fibrous conjunctive tissue proliferation, it was not statistically significant.

Important to emphasize that multinucleated giant cell, involved in the foreign body reaction, was not found in any ear analyzed. Similar fact was described by Rodríguez-Agirretxe et $a l .{ }^{9}$, when they analyzed histologically the eyeball of 42 rabbits after 30 days of PLGA implantation. In none of the cases was observed the encapsulation of the implant. According to Anderson et al..$^{13}$, the consequences of these reactions to the material surface can be devastating, which demonstrate the safety of this polymer.

Much remains to be done by biopolymers for pharmaceutical application. Therefore, constant improvement, new research and cooperation between related areas become paramount to the advancement in the manufacture of materials capable of been commercialized.

\section{Conclusion}

The tissue response by histological analysis of the mucosa of the tympanic cavity of guinea pigs, when receiving biodegradable implant, showed no statistically significant difference between ears with or without the implant.

\section{References}

1. Mikulec AA, Hartsock JJ, Salt AN. Permeability of the round window membrane is influenced by the composition of applied drug solutions and by common surgical procedures. Otol Neurotol. 2008 Oct;29(7):1020-6.

2. Plontke SK, Zimmermann R, Zenner H-P, Löwenheim H. Technical note on microcatheter implantation for local inner ear drug delivery: surgical technique and safety aspects. Otol Neurotol. 2006 Oct;27(7):912-7.

3. Kim ES, Gustenhoven E, Mescher MJ, Pararas EEL, Smith KA, Spencer AJ, Tandon V, Borenstein JT, Fiering J. A microfluidic reciprocating intracochlear drug delivery system with reservoir and active dose control. Lab Chip. 2014 Feb 21;14(4):710-21.

4. King EB, Salt AN, Kel GE, Eastwood HT, Leary SJO. Gentamicin administration on the stapes footplate causes greater hearing loss and vestibulotoxicity than round window administration in guinea pigs. Hear Res. 2013;304:159-66.

5. Liu H, Hao J, Li KS. Current strategies for drug delivery to the inner ear. Acta Pharm Sin B. 2013;3(2):86-96.

6. Fialho SL, Cunha Júnior AS. Drug delivery systems for the posterior segment of the eye: fundamental basis and applications. Arq Bras Oftalmol. 2007;70(1):173-9.

7. Perez AC, Cunha S, Fialho SL, Silva LM, Dorgam JV, Murushima AAB, Silva AR, Rossato M, Anselmo-Lima WT. Assessing the maxillary sinus mucosa of rabbits in the presence of biodegradable implants. Braz J Otorhinolaryngol. 2012;78(6):40-6.

8. Villanova JCO, Oréfice RL, Cunha AS. Aplicações Farmacêuticas de Polímeros. Polímeros Ciência e Tecnol. 2010;20(1):51-64.

9. Rodríguez-Agirretxe I, Vega SC, Rezola R, Vecino E, Mendicute J, Suarez-Cortes T, Acera A. The PLGA implant as an antimitotic delivery system after experimental trabeculectomy. Invest Ophthalmol Vis Sci. 2013;54(8):5227-35.

10. Ji W, Yang F, Seyednejad H, Chen Z, Hennink WE, Anderson JM, van den Beucken JJJP, Jansen JA. Biocompatibility and degradation characteristics of PLGA-based electrospun nanofibrous scaffolds with nanoapatite incorporation. Biomaterials. 2012 Oct;33(28):6604-14.

11. Williams DF. On the mechanisms of biocompatibility. Biomaterials. 2008 Jul;29(20):2941-53. 
Garcia LB et al.

12. Costa HO, Kato M, Sousa Neto OM, da Silva L, de Souza FC, de Castro MG. Evaluation of the middle ear tissue reaction to the presence of cellulose of bacteria. Experimental study in guinea pigs. Braz J Otorhinolaryngol. 2008;74(Suppl.1).

13. Anderson JM, Rodriguez A, Chang DT. Foreign body reaction to biomaterials. Semin Immunol. 2008;20(2):86-100.

\section{Correspondence:}

Leandro de Borborema Garcia

Rua Afonso Celso, 1102/74B

04119-061 São Paulo-SP Brasil

Tel.: (55 11)98637-0449

leandroborborema@yahoo.com.br

${ }^{1}$ Research performed at Institute of Advanced Sciences in Otolaryngology and Temporal Bone Microdissection Laboratory, Otology and Neurotology Discipline, Otolaryngology and Head and Neck Department, Federal University of Sao Paulo (UNIFESP), Brazil. 